\title{
Value of Contrast-Enhanced MRA versus Time-of-Flight MRA in Acute Ischemic Stroke MRI
}

\author{
(D)T. Boujan, (DU. Neuberger, (D). Pfaff, (D) S. Nagel, (D) C. Herweh, (D) M. Bendszus, and (D) M.A. Möhlenbruch
}

\begin{abstract}
BACKGROUND AND PURPOSE: Vessel imaging in acute ischemic stroke is essential to select patients with large-vessel occlusion for mechanical thrombectomy. Our aim was to compare the diagnostic accuracy of time-of-flight MR angiography and contrast-enhanced MR angiography for identification of vessel occlusion and collateral status in acute ischemic stroke.
\end{abstract}

MATERIALS AND METHODS: One hundred twenty-three patients with stroke with large-vessel occlusion before thrombectomy were included in this retrospective study. Before thrombectomy, 3T MR imaging, including conventional 3D TOF-MRA of the intracranial arteries and contrast-enhanced MRA of intra- and extracranial arteries, was performed. Both techniques were assessed independently by 2 neuroradiologists for location of the occlusion, imaging quality, and collateral status. Findings were compared, with subsequent DSA as the reference standard.

RESULTS: Both techniques had good interrater agreement of $\kappa=0.74(95 \% \mathrm{Cl}, 0.66-0.83)$ for TOF-MRA and $\kappa=0.72(95 \% \mathrm{Cl}, 0.63-0.80)$ for contrast-enhanced MRA. Occlusion localization differed significantly on TOF-MRA compared with DSA $(P<.001)$, while no significant difference was observed between DSA and contrast-enhanced MRA $(P=.75)$. Assessment of collaterals showed very good agreement between contrast-enhanced MRA and DSA (94.9\% with $P=.25)$, but only fair agreement between TOF-MRA and DSA $(23.2 \%$ with $P<.001)$.

CONCLUSIONS: Contrast-enhanced MRA offers better diagnostic accuracy than TOF-MRA in acute ischemic stroke. Contrast-enhanced MRA was superior in localizing vessel occlusion within a shorter acquisition time while providing a larger coverage, including extracranial vessels, and a more accurate assessment of collateral status. These results support inclusion of contrast-enhanced MRA in acute stroke MR imaging, perhaps making TOF-MRA superfluous.

ABBREVIATION: CE-MRA = contrast-enhanced MRA

$\mathrm{n}$ acute ischemic stroke, imaging plays a pivotal role in the ini-

tial diagnosis and treatment decisions. Especially in acute ischemic stroke due to large-vessel occlusion, vessel imaging, including CT angiography and MR angiography, is essential to select patients for thrombectomy as a highly effective treatment. ${ }^{1}$

Stroke MR imaging usually includes time-of-flight MRA for the detection of proximal vessel occlusion. ${ }^{2-4}$ However, TOFMRA has several major disadvantages: It provides only a small FOV, excluding extracranial vessels, and has a long acquisition time with the risk of motion artifacts $^{5}$ because the MR signal is generated by blood flow. ${ }^{6}$

Received April 15, 2018; accepted after revision June 17.

From the Departments of Neuroradiology (T.B., U.N., J.P., C.H., M.B., M.M.) and Neurology (S.N.), University of Heidelberg Medical Center, Heidelberg, Germany.

Timan Boujan and Ulf Neuberger contributed equally to this work.

Please address correspondence to Markus A. Möhlenbruch, MD, Department of Neuroradiology, University of Heidelberg Medical Center, Im Neuenheimer Feld 400, 69120 Heidelberg, Germany; e-mail:

markus.moehlenbruch@med.uni-heidelberg.de

http://dx.doi.org/10.3174/ajnr.A5771
Contrast-enhanced MR angiography (CE-MRA) with T1shortening paramagnetic contrast medium induces the MR signal predominantly unaffected by blood flow disturbances, while providing a larger coverage from the aortic arch up to intracranial arteries, ${ }^{7}$ and has a shorter acquisition time.

One of the most important questions in diagnostic imaging of acute stroke is, besides occlusion location as the principal indicator for further treatment, the assessment of pial collateral circulation as an independent predictor of outcome..$^{8-10}$

Recently, TOF-MRA and CE-MRA have both been implemented in the evaluation of patients with acute stroke with promising results. ${ }^{11,12}$ However, evaluation of CE-MRA as a method to identify intracranial occlusions and to assess collateral circulation has not yet provided conclusive results. ${ }^{13,14}$ As more centers use MR imaging for triage (especially in the 6- to 24-hour time window), it is important to determine the best MR imaging protocol to assess proximal occlusion and collaterals.

The aim of this retrospective study was to assess the interrater agreement and diagnostic accuracy of TOF-MRA and CE-MRA in 
Table 1: Acquisition parameters for MRA sequences

\begin{tabular}{lcc}
\hline \multicolumn{1}{c}{ Parameter } & CE-MRA & 3D TOF-MRA \\
\hline Receive coil & 12-Channel head and & 12-Channel head and \\
& neck coil combination & neck coil combination \\
TR/TE (ms) & $3.28 / 1.23$ & $22 / 3.83$ \\
Flip angle & $33^{\circ}$ & $18^{\circ}$ \\
Acquisition plane & Coronal & Axial \\
FOV (mm) & $300 \times 300$ & $200 \times 200$ \\
Reconstructed voxel size (mm) & $0.9 \times 0.7 \times 0.8$ & $0.7 \times 0.5 \times 0.6$ \\
Slice oversampling (\%) & 9.1 & 20 \\
No. of slices per slab & 88 & 40 \\
No. of slabs & 1 & 3 \\
Slice thickness (mm) & 0.8 & 0.64 \\
Partial Fourier (phase and slice directions) & $7 / 8$ and $6 / 8$ & $6 / 8$ and $6 / 8$ \\
Total acquisition time & $64 \mathrm{sec}$ & $3 \mathrm{~min} 7 \mathrm{sec}$ \\
\hline
\end{tabular}

after arrival of contrast media in the proximal common carotid artery. The second bolus of contrast (PWI) was identical, with injection of a volume of $0.1-\mathrm{mmol} / \mathrm{kg}$ of body weight bolus of gadoterate meglumine, $0.5 \mathrm{mmol} / \mathrm{mL}$ intravenously at $3.5 \mathrm{~mL} / \mathrm{s}$ with an automatic power injector, followed by a 20-mL saline flush.

Source images and 3D maximum-intensity-projection images of CE-MRA and TOF-MRA were generated.

\section{DSA Imaging}

Diagnostic DSA before thrombectomy identifying the location of occlusions and assessing the status of collaterals in the acute phase of ischemic stroke, compared with the reference standard DSA, in patients with large-vessel occlusion eligible for thrombectomy.

The hypothesis of this study was that CE-MRA is superior to TOF-MRA in determining the site of occlusion as the key element of acute stroke MR imaging before thrombectomy. Moreover, we hypothesized that CE-MRA offers better diagnostic assessment of collateral status. Additionally, by covering a larger FOV, including the supraaortic vessels, CE-MRA offers the possibility of additional findings.

\section{MATERIALS AND METHODS \\ Patient Selection}

Due to the retrospective character of the study, individual written informed consent was waived by the institutional review board. One hundred twenty-three patients were prospectively included in a data base between January 2011 and July 2015.

Inclusion criteria for this study were the following: 1) clinical symptoms suggestive of acute stroke due to large-vessel occlusion, 2) DSA within 60 minutes after stroke MR imaging for thrombectomy, and 3) stroke MR imaging, including TOF- and CE-MRA, with the absence of motion artifacts impeding assessment of intracranial vessel occlusion.

\section{MR Imaging}

All examinations were performed on a 3T MR imaging scanner (Magnetom Trio or Verio; Siemens, Erlangen, Germany) using a 12-channel head and neck coil array.

The complete acute stroke MR imaging protocol included parenchymal brain imaging sequences (axial DWI, T2 FLAIR, and SWI), angiographic sequences without contrast media (3D TOF), angiographic sequences with injection of a first bolus of contrast (CE-MRA), and perfusion-weighted imaging with the injection of a second bolus of contrast. The total average acquisition time for all MR imaging sequences was 17 minutes. The MRA acquisition parameters are provided in Table 1.

A volume of a $0.1-\mathrm{mmol} / \mathrm{kg}$ of body weight bolus of gadoterate meglumine, $0.5 \mathrm{mmol} / \mathrm{mL}$, (Dotarem; Guerbet, Aulnay-sousBois, France) was administered via a peripheral venous catheter at $2 \mathrm{~mL} / \mathrm{s}$ with an automatic power injector, followed by a $20-\mathrm{mL}$ saline flush, with a bolus-tracking acquisition for the CE-MRA. A bolus-tracking sequence was started simultaneously with the injection. The image-acquisition sequence was launched manually was performed as the standard reference for extra- and intracranial artery analysis. All DSA examinations were performed by experienced neurointerventionalists on a biplanar system (Artis zee biplane; Siemens). Angiographic images were acquired at 4 frames per second with a manual injection of iodinated contrast media.

\section{Data Analysis}

The TOF-MRA and CE-MRA sequences (MPR and MIP images) were evaluated independently and in random order on a commercially available 3D workstation by 2 neuroradiologists (T.B. and U.N.) with 8 and 2 years of experience, respectively. Results were compared with those of diagnostic DSA images in terms of occlusion location, quality of imaging, relevant vascular findings of supra-aortic vessels in CE-MRA, and assessment of collaterals. Readings occurred for several days, and cases were randomly assigned to prevent recall. Potential disagreements were discussed to reach a consensus.

The overall MR image quality was analyzed with a subjective interpretation score using a 3 -point scoring scale: $0=$ poor quality with a substantial number of artifacts, interpretation not possible; 1 = moderate quality with a mild-to-moderate number of artifacts, noise not interfering with diagnosis/interpretation; 2 = good/excellent image quality with no-to-minimal artifacts.

Occlusion location was defined as the proximal M1 segment (first half of the M1 segment), distal M1 segment (second half of the M1 segment), M2 segment, M3 segment, proximal internal carotid artery, distal ICA (subdivided into carotid-I, -L, and -T occlusions according to Liebeskind et $\mathrm{al}^{15}$ ), common carotid artery, and basilar artery.

Collaterals were evaluated according to a simplified 3-point scale based on the 4-point CT scale by Tan et al, ${ }^{16}$ comparing the vascularity distal to the occlusion between the ischemic and the healthy hemisphere: $0=$ no collaterals (no filling of the occluded area), $1=$ poor collaterals ( $>0 \%$ but $\leq 50 \%$ filling of the occluded area), $2=$ moderate/good collaterals $(>50 \%$ filling of the occluded area).

Finally, we evaluated the relevant vascular findings of supraaortic vessels, which may have influenced the choice of guiding or distal-access catheter (eg, vulnerable aortic arch and vascular variants). 


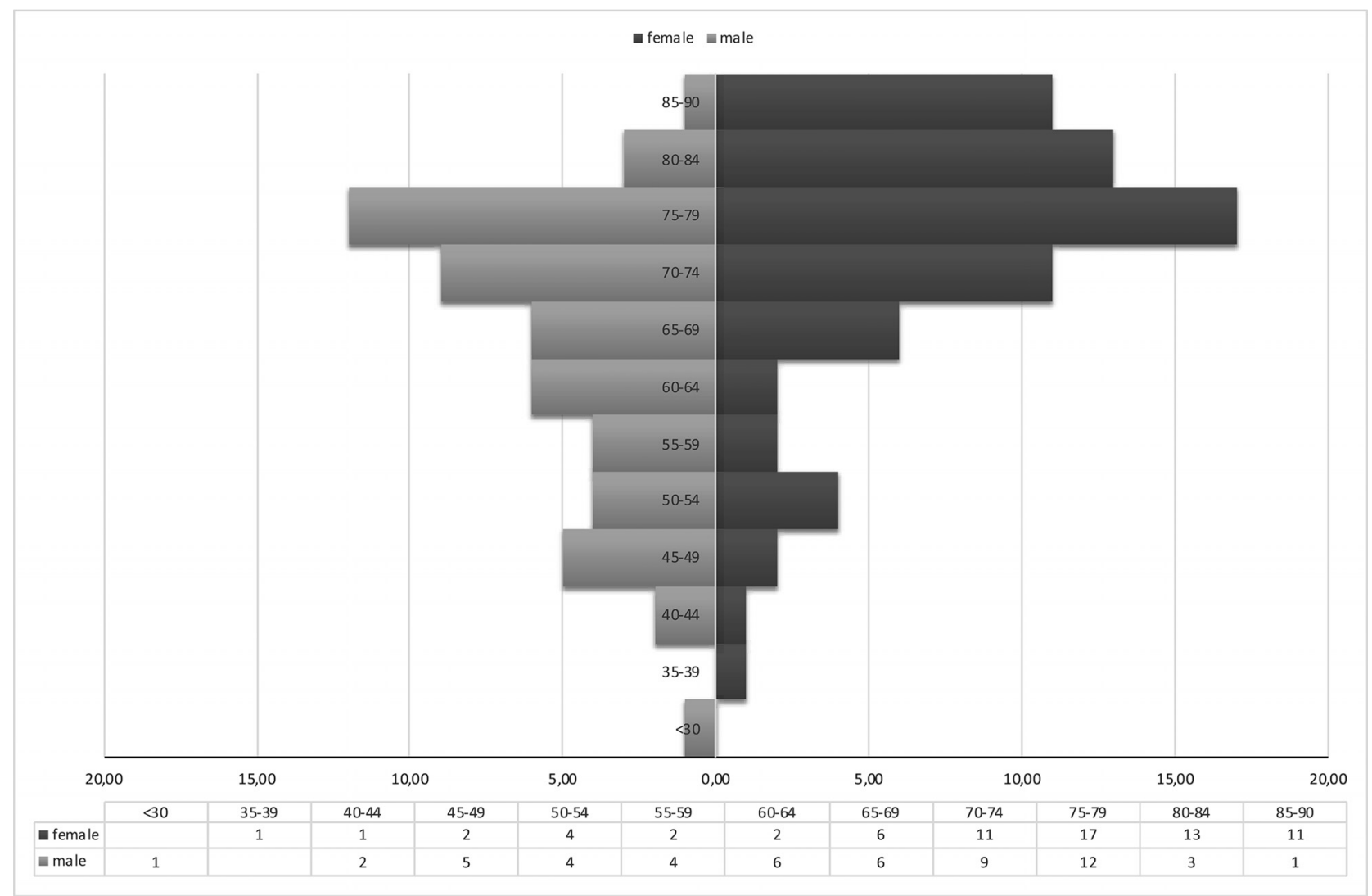

FIG 1. Sex and age distribution (in absolute numbers).

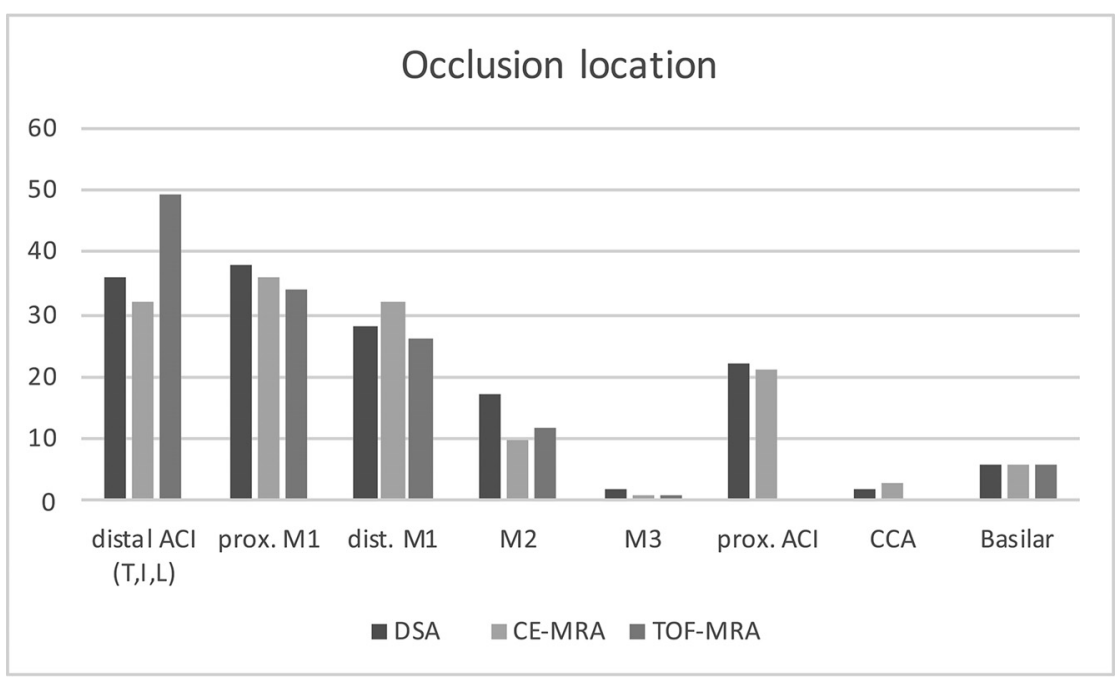

FIG 2. Site of occlusion (absolute numbers) based on the respective imaging technique.

\section{Statistical Analysis}

Statistical analysis was performed using GraphPad Prism, Version 7 (GraphPad Software, San Diego, California) and Excel (Microsoft, Redmond, Washington). Interrater agreement was determined using the Cohen $\kappa$ coefficient. Agreement was graded according to Altman's definition ${ }^{17}$ with $\kappa=0.0-0.20$, poor; $\kappa=$ $0.21-0.40$, fair; $\kappa=0.41-0.60$, moderate; $\kappa=0.61-0.80$, good; and $\kappa=0.81-1.00$, very good.

Measurements (occlusion location, collaterals) based on the 2 MRA sequences were compared with the reference imaging stan- dard DSA using a Wilcoxon signed rank test. A variable was considered statistically significant only if $P<.05$. $^{18}$

\section{RESULTS}

\section{Patient Selection}

Overall, 123 patients met the inclusion criteria (mean age, $70.3 \pm 12.9$ years; 70 women, 53 men) between January 2011 and July 2015. Sex and age distributions are given in Fig 1.

\section{Evaluation of Image Quality}

Only $3.25 \%(4 / 123)$ of all TOF-MRAs and $1.62 \%(2 / 123)$ of all CE-MRAs could not be interpreted because of motion artifacts. The imaging quality was not statistically different between TOFMRA (mean, $1.72 \pm 0.49$ ) and CE-MRA (mean, $1.83 \pm 0.45$ ) using the 3-step scoring scale. Interrater agreement for assessment of imaging quality was moderate for both CE-MRA $(0.44$; 95\% CI, $0.25-$ $0.64)$ and TOF-MRA $(0.51 ; 95 \%$ CI, 0.35-0.68).

\section{Occlusion Location}

In all 123 patients, an arterial occlusion was identifiable in 3D TOF and CE-MRA sequences. Interrater agreement for occlusion location was good for CE-MRA $(\kappa=0.73$; $95 \% \mathrm{CI}, 0.66-0.82)$ and TOF-MRA $(\kappa=0.74 ; 95 \% \mathrm{CI}, 0.66-0.83)$. 
Table 2: Identification of intracranial occlusion location with 3D TOF-MRA and CE-MRA compared with DSA as the reference standard

\begin{tabular}{lccc}
\hline Occlusion Location & TOF-MRA & CE-MRA & DSA \\
\hline CCA & $0(0 \%)$ & $3(66.7 \%)$ & 2 \\
Proximal ICA & $0(0 \%)$ & $21(90.5 \%)$ & 21 \\
Distal ICA & $53(18.9 \%)$ & $34(100 \%)$ & 36 \\
M1 & $54(96.3 \%)$ & $68(94.1 \%)$ & 66 \\
M2 & $14(92.9 \%)$ & $13(92.3 \%)$ & 17 \\
M3 & $1(100 \%)$ & $1(100 \%)$ & 2 \\
Basilar artery & $6(100 \%)$ & $6(100 \%)$ & 6 \\
\hline
\end{tabular}

Note:-CCA indicates common carotid artery.

a Site of occlusion (absolute numbers), based on the respective imaging modality with percentage of agreement with DSA.

Table 3: Cases with discrepancies in occlusion location between 3D TOF-MRA and CE-MRA compared with DSA as the reference standard $^{\mathrm{a}}$

\begin{tabular}{|c|c|c|c|}
\hline Patient No. & TOF-MRA & CE-MRA & DSA \\
\hline 2 & 1,3 & 6,3 & 6,3 \\
\hline 10 & 2 & 3 & 3 \\
\hline 11 & 1,2 & 7,2 & 6,2 \\
\hline 12 & 1 & 6,2 & 6,2 \\
\hline 16 & 1,2 & 6,2 & 6,2 \\
\hline 19 & 1 & 6,2 & 6,2 \\
\hline 23 & 1 & 6,3 & 6,3 \\
\hline 30 & 1 & 6,1 & 1 \\
\hline 34 & 1 & 7,1 & 7,1 \\
\hline 42 & 1 & 6 & 6 \\
\hline 59 & 1 & 6 & 6,4 \\
\hline 62 & 1,3 & 6,3 & 6,3 \\
\hline 65 & 4 & 3 & 4 \\
\hline 66 & 1 & 6,2 & 6,2 \\
\hline 70 & 1 & 6,1 & 6,1 \\
\hline 71 & 1 & 6,2 & 6,2 \\
\hline 79 & 1 & 6,1 & 6,1 \\
\hline 80 & 1,3 & 6,2 & 6,2 \\
\hline 84 & 1 & 7 & 7,1 \\
\hline 87 & 1,2 & 6,2 & 6,2 \\
\hline 94 & 1 & 6,2 & 6,2 \\
\hline 97 & 1 & 6,2 & 1 \\
\hline 98 & 1,2 & 6,2 & 6,2 \\
\hline 107 & 1 & 6,1 & 6,1 \\
\hline 117 & 1 & 6,1 & 6,1 \\
\hline 118 & 4 & 4 & 5 \\
\hline 119 & 1 & 6,1 & 6,1 \\
\hline 120 & 1 & 6,2 & 6,2 \\
\hline
\end{tabular}

${ }^{a}$ Occlusion location: $1=$ distal ICA; $2=$ proximal Ml; $3=\operatorname{distal} \mathrm{Ml} ; 4=\mathrm{M} 2 ; 5=\mathrm{M} 3$; $6=$ proximal ICA; $7=$ CCA.

Specificity and sensitivity for detecting the correct occlusion site compared with DSA were $92.0 \%$ and $91.7 \%$ for TOF-MRA and $99.0 \%$ and $92.0 \%$ for CE-MRA, respectively. Sensitivity for detecting a distal ICA occlusion was similar for both techniques (100\% for TOF-MRA versus $94 \%$ for CE-MRA), whereas specificity was better for CE-MRA (100\% versus $75 \%$ for TOF-MRA).

A Wilcoxon signed rank test revealed significant differences in the occlusion location between TOF-MRA and DSA $(P<.001)$, whereas there was no statistically significant difference between CE-MRA and DSA $(P=.75)$.

The occlusion locations are presented in Fig 2 and Table 2. The cases in which there were discrepancies among TOF MRA, CE MRA, and DSA are listed in Table 3. Imaging examples are shown in Fig 3.

\section{Evaluation of Collaterals}

The assessment of collaterals is more sensitive to motion artifacts compared with occlusion location of proximal vessels because peripheral vessels/collaterals are much smaller; therefore, 3.25\% (4/123) of all TOF-MRAs and 5.69\% (7/123) of all CE-MRAs could not be interpreted. We only compared collaterals for M1 and M2 occlusions because pial collaterals could be retrogradely filled vessels from the ipsilateral anterior cerebral artery, posterior communicating artery, or lenticulostriate arteries. Because the assessment of collateral status was not possible for occlusions of the ICA and basilar artery, we excluded these.

Furthermore, the assessment of the collaterals on DSA was impossible in $9.7 \%$ (7/72) of the remaining cases because of the short series duration or lack of ipsilateral A1 or posterior communicating arteries. Therefore, the collateral score could be compared in 65 patients.

We analyzed the collateral status in the reference standard DSA with a very good interrater agreement with $\kappa=0.92(95 \%$ CI, 0.80-0.99). Interrater agreement for assessing the collaterals was considered good for CE-MRA with $\kappa=0.70$ (95\% CI, 0.560.85 ) and moderate for TOF-MRA with $\kappa=0.40$ (95\% CI, 0.19$0.62)$. Imaging examples are given in Fig 3.

The collateral score was identical between DSA and CE-MRA in $87.7 \%$ of cases $(57 / 65)$ with no statistically significant difference $(P=.125)$, while there was a significant difference in collateral assessment between TOF-MRA and DSA $(P<.001)$ and only $21.5 \%(14 / 65)$ of cases were assigned correctly. Imaging examples are shown in Fig 4.

\section{Relevant Vascular Findings of Supra-Aortic Vessels}

CE-MRA demonstrated relevant vascular findings of the supraaortic vessels, which were decisive for the endovascular treatment planning in $18.7 \%(23 / 123)$ of all patients. The relevant vascular findings are presented in Table 4.

\section{DISCUSSION}

Both TOF-MRA ${ }^{19}$ and CE-MRA ${ }^{20-22}$ are frequently used and sensitive techniques for the triage of patients with acute stroke. Traditionally, TOF-MRA was primarily used to detect intracranial occlusions and occlusions in proximity to the skull base, while CE-MRA was usually performed to provide anatomic information from the aortic arch up to the skull base.

The advancement of stronger magnetic fields (ie, 3T), optimized sequences, and better receive coil arrays made it feasible to visualize intracranial cerebral arteries by CE-MRA at sufficient resolution. However, for more than a decade, multimodal CT has established itself as a less expensive and accessible alternative in the emergency assessment of patients with stroke, especially for patients with a short time window. ${ }^{23}$ With the steady progress of neurointerventional therapy during recent years, mechanical thrombectomy has proved to be a highly effective therapy option, ${ }^{24}$ well beyond the traditional time window of 6 hours, and up to 24 hours in eligible patients. ${ }^{11,12}$ These recent developments stress the importance of MR imaging in acute stroke triage, especially in the 6- to 24-hour time window and necessitate determining the best MR images for assessment of the occlusion site and collateral status. 

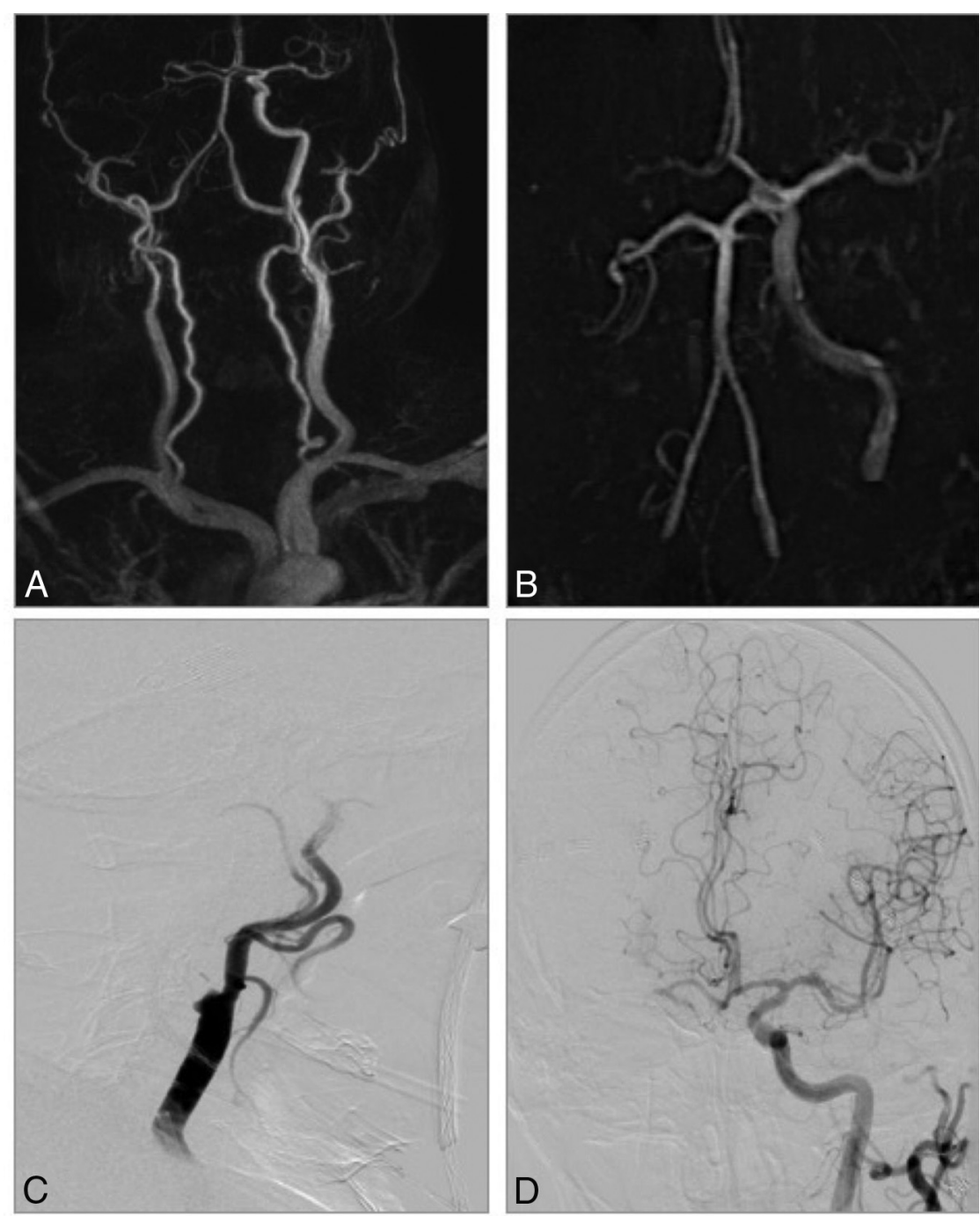

FIG 3. CE-MRA and TOF-MRA in an 81-year-old man with acute stroke symptoms before treatment. A, CE-MRA shows a tandem occlusion of the proximal ICA and right MI segment with poor collaterals. $B$, TOF-MRA shows an occlusion of distal ICA (carotid-L). $C$ and $D$, DSA confirms the tandem occlusion and CE-MRA diagnosis.

The present study indicates the superiority of CE-MRA in detecting the intracranial vessel occlusion and assessing intracranial collateral status, while simultaneously providing larger coverage at a shorter acquisition time compared with TOF-MRA.

The primary differences between TOF-MRA and DSA were found in patients with occlusions of the distal supraclinoid ICA (occlusions of the carotid-I, $-\mathrm{L}$, and $-\mathrm{T}^{15}$ ) and proximal ICA. This difference is mostly due to distal occlusions mimicking proximal extracranial ICA occlusions ("pseudo-occlusion") and proximal occlusions mimicking distal intracranial ICA occlusions. This misapprehension could be a result of the nonenhanced nature of TOF-MRA, a technique that depends on the flow velocity and direction of blood. Previous studies have already reported this limitation of TOF-MRA when assessing these occlusion sites. ${ }^{25-27}$ The better accuracy of CEMRA was not dependent on image quality, which was not significantly different for the 2 techniques despite TOF-MRA having better spatial resolution. The better accuracy of CE-
MRA, on the other hand, might be due to the T1-shortening effect of gadolinium. The effect compensates for the signal loss caused by spin dephasing occurring in TOF-MRA, ${ }^{19}$ especially when the arterial flow is slow or oriented parallel to the section plane, which applies to imaging of the M2 segment. In these cases, TOF-MRA may indicate an incorrect occlusion site, with the level of occlusion more proximal than expected.

Similarly, for the assessment of collaterals as independent predictors of outcome,${ }^{8-10}$ CE-MRA was not affected by the signal loss observed in TOFMRA. This particular signal loss might be due to low flow in vessels distal to the occlusion. Furthermore, slow collateral flow over leptomeningeal connections cannot be visualized on TOF-MRA. ${ }^{28}$ This issue resulted in CE-MRA being significantly more accurate than TOFMRA in assessing collaterals compared with the criterion standard of DSA. A previously performed study with $44 \mathrm{pa}-$ tients found similar results with CEMRA, but not TOF-MRA, being a reliable predictor of infarct outcome in patients with stroke with proximal arterial occlusion of the anterior circulation using visual scoring. ${ }^{29}$ The predictive performance could be increased by applying an automated atlas-based collateral assessment.

Besides assessment of the occlusion site and collaterals, CE-MRA offered additional advantages that could not be compared in the present study due to the limited FOV on TOF-MRA. CE-MRA showed relevant vascular findings of the supra-aortic vessels in $18.7 \%$ of patients, which are crucial for the planning of endovascular treatment. The choice of a guiding or distal-access catheter as well as selection of the vessel providing better access (in case of access from the vertebral artery) may be influenced by these findings as well as a priori knowledge of the internal carotid artery condition (eg, occlusion, site of occlusion, pseudo-occlusion).

\section{Limitations of CE-MRA}

In comparison with TOF-MRA, CE-MRA requires more preparation with the following: 1) filling a power injector with contrast media, and 2) a sequence for bolus-tracking that takes additional time during stroke MR imaging (64 seconds). Currently, the use of contrast media is controversial due to potential risk of nephrogenic systemic fibrosis, in particular with unknown renal retention parameters, ${ }^{30,31}$ and possible brain gadolinium deposition. ${ }^{32}$ This risk is especially the case when not performing bolus PWI but instead using an arterial spin-labeling perfusion. Both risks 

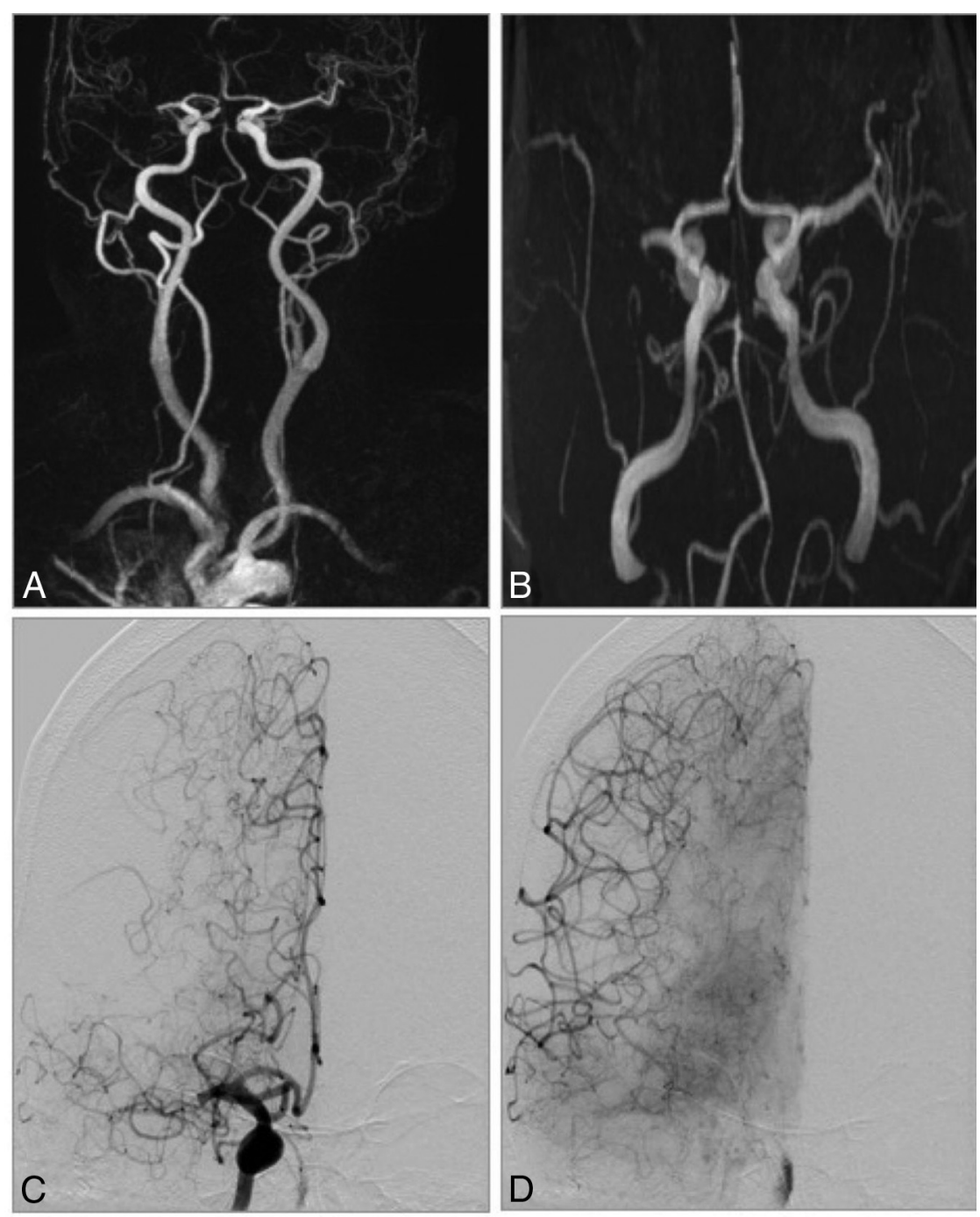

FIG 4. CE-MRA and TOF-MRA in a 72-year-old woman with acute stroke symptoms before treatment. $A, C E-M R A$ shows an occlusion of right M1 segment with good collaterals. $B$, TOF-MRA shows an occlusion of the $\mathrm{Ml}$ segment and poor collaterals. $C$ and D, DSA shows a right-sided MI occlusion with good collaterals as in the CE-MRA.

\section{Table 4: Relevant vascular findings of supra-aortic vessels}

\begin{tabular}{lc}
\hline \multicolumn{1}{c}{ Relevant Findings in CE-MRA } & No. (\%) \\
\hline Vulnerable aortic arch (type III with at least 1 & $11(8.9)$ \\
cervical vessel originating below the inferior & \\
margin of the aortic arch as described by & \\
Demertzis ${ }^{35}$ ) and a vascular variant & \\
(eg, severe vessel elongation) & $2(1.6)$ \\
Dissection of the ipsilateral ICA & $5(4.1)$ \\
PICA termination of hypoplastic vertebral artery, & \\
right or left, in case of basilar artery thrombosis & \\
Relevant ipsilateral ICA stenosis in case of M1-M2 & $5(4.1)$ \\
thrombosis & \\
\hline
\end{tabular}

can be minimized using macrocyclic gadolinium-based contrast agents. $^{33}$

Furthermore, in our subjective experience, the first bolus of contrast media for CE-MRA did not affect the subsequent PWI analysis and was not a major limiting factor for the diagnostic interpretation, as was shown before. ${ }^{34}$

CE-MRA has lower spatial resolution compared with TOFMRA, which could lead to poorer performance in addressing future prospective trials. more precise characteristics of the clot, even if the localization is known.

\section{Study Limitations}

The main limitation of this study is its retrospective design and the inclusion of nonconsecutive patients. However, the data were collected in a prospective data base. Moreover, a precise assessment of collateral status in DSA is only possible with 3-vessel angiography. Nevertheless, we performed only 1 injection at the site of occlusion to reach the clot as soon as possible and to avoid treatment delay. Another potential source of bias in this study might be the 30- to 60-minute delay between MRA examinations and DSA, with potential alterations of the findings (eg, thrombus migration, especially after intravenous thrombolysis). Moreover, the subjective assessment of image quality and collaterals, which is more sensitive to motion artifacts, may be a further limitation of the study.

\section{CONCLUSIONS}

Our study indicates that CE-MRA is superior to TOF-MRA in identifying occlusion location and assessing the status of collaterals in patients with ischemic stroke, with shorter examination times. Moreover, CE-MRA can provide crucial information for the planning of endovascular treatment by covering a larger FOV. The inclusion of supra-aortic vessels, for example, can inform the selection of the appropriate guiding or distal-access catheters. These findings indicate that CE-MRA could replace TOF-MRA in the triage of patients with acute stroke and its use should be evaluated in

Disclosures: Johannes Pfaff-UNRELATED: Travel/Accommodations/Meeting Expenses Unrelated to Activities Listed: Stryker, MicroVention. Simon NagelUNRELATED: Consultancy, Brainomix, Boehringer Ingelheim; Payment for Lectures Including Service on Speakers Bureaus: Medtronic, Bayer AG, Pfizer; Travel/Accommodations/Meeting Expenses Unrelated to Activities Listed: Brainomix. Christian Herweh—UNRELATED: Consultancy: Brainomix; Payment for Lectures Including Service on Speakers Bureaus: Medtronic, Bristol-Myers Squibb; Travel/Accommodations/Meeting Expenses Unrelated to Activities Listed: Stryker. Martin Bendszus - UNRELATED: Board Membership: Data and Safety Monitoring Board for Vascular Dynamics, Guerbet, Boehringer Ingelheim; Consultancy: Codman, Roche, Guerbet, Boehringer Ingelheim, B Braun; Grants/Grants Pending: Deutsche Forschungsgemeinschaft, Hopp Foundation, Novartis, Siemens, Guerbet, Stryker, Covidien, European Union*; Payment for Lectures Including Service on Speakers Bureaus: Novartis, Roche, Guerbet, Teva Pharmaceutical Industries, Bayer AG, Codman. Markus A. Möhlenbruch—UNRELATED: Board Membership: Codman; Consultancy: Medtronic, MicroVention, Stryker; Grants/Grants Pending: Balt, MicroVention*; Payment for Lectures Including Service on Speakers Bureaus: Medtronic, MicroVention, Stryker. *Money paid to institution. 


\section{REFERENCES}

1. Saver JL, Goyal M, van der Lugt A, et al; HERMES Collaborators. Time to treatment with endovascular thrombectomy and outcomes from ischemic stroke: a meta-analysis. JAMA 2016;316:1279-88 CrossRef Medline

2. Nagel S, Schellinger PD, Hartmann M, et al. Therapy of acute basilar artery occlusion: intraarterial thrombolysis alone vs bridging therapy. Stroke 2009;40:140-46 CrossRef Medline

3. Barlinn K, Alexandrov AV. Vascular imaging in stroke: comparative analysis. Neurotherapeutics 2011;8:340-48 CrossRef Medline

4. Stock KW, Radue EW, Jacob AL, et al. Intracranial arteries: prospective blinded comparative study of MR angiography and DSA in 50 patients. Radiology 1995;195:451-56 CrossRef Medline

5. Miyazaki M, Lee VS. Nonenhanced MR Angiography. Radiology 2008;248:20-43

6. Bash S, Villablanca JP, Jahan R, et al. Intracranial vascular stenosis and occlusive disease: evaluation with CT angiography, MR angiography, and digital subtraction angiography. AJNR Am J Neuroradiol 2005;26:1012-21 Medline

7. Yang CW, Carr JC, Futterer SF, et al. Contrast-enhanced MR angiography of the carotid and vertebrobasilar circulations. AJNR Am J Neuroradiol 2005;26:2095-101 Medline

8. Cheng-Ching E, Frontera JA, Man S, et al. Degree of collaterals and not time is the determining factor of core infarct volume within 6 hours of stroke onset. AJNR Am J Neuroradiol 2015;36:1272-76 CrossRef Medline

9. Kluytmans M, van der Grond J, van Everdingen KJ, et al. Cerebral hemodynamics in relation to patterns of collateral flow. Stroke 1999;30:1432-39 CrossRef Medline

10. Bang OY, Saver JL, Kim SJ, et al. Collateral flow predicts response to endovascular therapy for acute ischemic stroke. Stroke 2011;42: 693-99 CrossRef Medline

11. Albers GW, Marks MP, Kemp S, et al; DEFUSE 3 Investigators. Thrombectomy for stroke at 6 to 16 hours with selection by perfusion imaging. N Engl J Med 2018;378:708-18 CrossRef Medline

12. Nogueira RG, Jadhav AP, Haussen DC, et al; DAWN Trial Investigators. Thrombectomy 6 to 24 hours after stroke with a mismatch between deficit and infarct. $N$ Engl J Med 2018;378:11-21 CrossRef Medline

13. Hernández-Pérez M, Puig J, Blasco G, et al. Dynamic magnetic resonance angiography provides collateral circulation and hemodynamic information in acute ischemic stroke. Stroke 2016;47:531-34 CrossRef Medline

14. Martinon E, Lefevre PH, Thouant $\mathrm{P}$, et al. Collateral circulation in acute stroke: assessing methods and impact-a literature review. I Neuroradiol 2014:41:97-107 CrossRef Medline

15. Liebeskind DS, Flint AC, Budzik RF; MERCI and Multi-MERCI Investigators. Carotid I's, L's and T's: collaterals shape the outcome of intracranial carotid occlusion in acute ischemic stroke. J Neurointerv Surg 2015;7:402-07 CrossRef Medline

16. Tan IYL, Demchuk AM, Hopyan J, et al. CT angiography clot burden score and collateral score: correlation with clinical and radiologic outcomes in acute middle cerebral artery infarct. AJNR Am J Neuroradiol 2009;30:525-31 CrossRef Medline

17. Altman DG. Practical Statistics for Medical Research. Chapman \& Hall/CRC; 1990

18. Rosner B, Glynn RJ, Lee ML. The Wilcoxon signed rank test for paired comparisons of clustered data. Biometrics 2006;62:185-92 CrossRef Medline

19. Yang JJ, Hill MD, Morrish WF, et al. Comparison of pre- and postcontrast 3D time-of-flight MR angiography for the evaluation of distal intracranial branch occlusions in acute ischemic stroke. AJNR Am J Neuroradiol 2002;23:557-67 Medline

20. Nael K, Khan R, Choudhary G, et al. Six-minute magnetic resonance imaging protocol for evaluation of acute ischemic stroke: pushing the boundaries. Stroke 2014;45:1985-91 CrossRef Medline

21. Phan T, Huston J 3rd, Bernstein MA, et al. Contrast-enhanced magnetic resonance angiography of the cervical vessels: experience with 422 patients. Stroke 2001;32:2282-86 CrossRef Medline

22. Le Bras A, Raoult H, Ferré JC, et al. Optimal MRI sequence for identifying occlusion location in acute stroke: which value of time-resolved contrast-enhanced MRA? AJNR Am J Neuroradiol 2015;36: 1081-88 CrossRef Medline

23. Meissner W, Sibon I, Rouanet F, et al. MRI versus CT in acute stroke. Lancet 2007;369:1342 Medline

24. Goyal M, Menon BK, van Zwam WH, et al; HERMES collaborators Endovascular thrombectomy after large-vessel ischaemic stroke: a meta-analysis of individual patient data from five randomised trials. Lancet 2016;387:1723-31 CrossRef Medline

25. Saager C, Fitting T, Goebell E, et al. Contrast-enhanced MR angiography improves detection of carotid-T occlusion by acute stroke MRI. Clin Neuroradiol 2008;18:163-77

26. Igase K, Igase M, Matsubara I, et al. Mismatch between TOF MR angiography and $\mathrm{CT}$ angiography of the middle cerebral artery may be a critical sign in cerebrovascular dynamics. Yonsei Med J 2018;59: 80-84 CrossRef Medline

27. Pedraza S, Silva Y, Mendez J, et al. Comparison of preperfusion and postperfusion magnetic resonance angiography in acute stroke. Stroke 2004;35:2105-10 CrossRef Medline

28. Heiserman JE, Drayer BP, Keller PJ, et al. Intracranial vascular stenosis and occlusion: evaluation with three-dimensional time-offlight MR angiography. Radiology 1992;185:667-73 CrossRef Medline

29. Ernst M, Forkert ND, Brehmer L, et al. Prediction of infarction and reperfusion in stroke by flow- and volume-weighted collateral signal in MR angiography. AJNR Am J Neuroradiol 2015;36:275-82 CrossRef Medline

30. Cowper SE, Robin HS, Steinberg SM, et al. Scleromyxoedema-like cutaneous diseases in renal-dialysis patients. Lancet 2000;356: 1000-01 CrossRef Medline

31. Thomsen HS, Webb JA, eds. Contrast Media: Safety Issues and ESUR Guidelines. Berlin Heidelberg: Springer Science \& Business Media; 2014

32. Kanda T, Ishii $\mathrm{K}$, Kawaguchi $\mathrm{H}$, et al. High signal intensity in the dentate nucleus and globus pallidus on images: relationship with increasing cumulative dose of a gadolinium-based contrast material. Radiology 2014;270:834-41 CrossRef Medline

33. Radbruch A, Weberling LD, Kieslich PJ, et al. Gadolinium retention in the dentate nucleus and globus pallidus is dependent on the class of contrast agent. Radiology 2015;275:783-91 CrossRef Medline

34. Nael K, Meshksar A, Ellingson B, et al. Combined low-dose contrastenhanced MR angiography and perfusion for acute ischemic stroke at 3T: a more efficient stroke protocol. AJNR Am J Neuroradiol 2014; 35:1078-84 CrossRef Medline

35. Demertzis S, Hurni S, Stalder M, et al. Aortic arch morphometry in living humans. J Anat 2010;217:588-96 CrossRef Medline 